Please do not remove this page

RMIT

UNIVERSITY

\title{
Determining the influence of population variation on compliance with radiofrequency exposure limits: proposed study
}

Sauren, Maia; McKenzie, Raymond; Cosic, Irena

https://researchrepository.rmit.edu.au/esploro/outputs/9921859227401341/filesAndLinks?institution=61RMIT_INST\&index=null

Sauren, M., McKenzie, R., \& Cosic, I. (2005). Determining the influence of population variation on compliance with radiofrequency exposure limits: proposed study. Proceedings of the 27th Annual International Conference of the IEEE Engineering in Medicine and Biology Society.

https://doi.org/10.1109/IEMBS.2005.1617095

Published Version: https://doi.org/10.1109/IEMBS.2005.1617095

Repository homepage: https://researchrepository.rmit.edu.au

(c) 2005 IEEE. Personal use of this material is permitted. However, permission to reprint/republish this material for advertising or promotional purposes or for creating new collective works for resale or redistribution to servers or lists, or to reuse any copyrighted component of this work in other works must be obtained from the IEEE.

Downloaded On 2023/04/27 00:06:28 +1000 


\title{
Determining The Influence Of Population Variation On Compliance With Radiofrequency Exposure Limits: Proposed Study
}

\author{
Maia Sauren, Ray J. McKenzie, and Irena Cosic, Member, IEEE
}

\begin{abstract}
Currently, compliance with safety limits for human exposure to radiofrequency (RF) fields is demonstrated by methods that rely on certain assumptions and approximations, which include among other things, human anatomical features, tissue types and the dielectric properties of these tissues. This paper reviews some of the available data and outlines a proposal for an encompassing study to investigate which of these assumptions are appropriate; what approximation can be used in physical and computational modeling of humans for specific energy absorption rate (SAR) calculations (a key compliance metric); and what trade-offs can be made between accuracy and modeling requirements for practical considerations. Key issues to be investigated are how SAR varies between children and adults, between males and females, and how to model SAR in the fetus of pregnant females. It is hoped that the proposed study will produce models and methods which allow for faster, more accurate and more efficient compliance with radiofrequency exposure limits.
\end{abstract}

\section{INTRODUCTION}

$\mathrm{I}$ is difficult to compare studies regarding energy absorption in humans from various radiofrequency (RF) sources due to the contradictions and inconsistencies in RF dosimetry literature. Ranging assumptions, models and methodology in the available literature mean that firm conclusions may not be drawn. Assumptions used in Specific Absorption Rate (SAR) studies (a key metric in determining compliance with safety limits for human exposure to RF) have been insufficiently explored.

Compliance with RF exposure limits is typically demonstrated by measurement or calculation of the SAR produced in simplified physical or computational models exposed to the RF source in question. Physical measurements are performed using a robot-controlled measuring system and a fiberglass head- or body- shaped phantom filled with tissue-simulating dielectric equivalent

Manuscript submitted 8 April, 2001. This work was supported by the National Australian Centre of Radiofrequency Bioeffects Research (ACRBR).

M. Sauren is with School of Electrical and Computer Engineering, RMIT University, GPO Box 2476V, Melbourne 3001 VIC Australia and with Australian Centre of Radiofrequency Bioeffects Research (ACRBR), Australia (phone: +61 3 9253-6356; fax: +61 3 9925-2954; e-mail: maia.sauren@team.telstra.com).

R. J. McKenzie is with School of Electrical and Computer Engineering, RMIT University, GPO Box 2476V, Melbourne 3001 VIC Australia and with Australian Centre of Radiofrequency Bioeffects Research (ACRBR), Australia (e-mail: ray.mckenzie@team.telstra.com).

I. Cosic is with School of Electrical and Computer Engineering, RMIT University, GPO Box 2476V, Melbourne 3001 VIC Australia and with Australian Centre of Radiofrequency Bioeffects Research (ACRBR), Australia (e-mail: irena.cosic@rmit.edu.au). liquid. SAR is determined by the robot by measuring internal electrical field during RF exposure of the phantom. Physical models are necessarily much simplified (containing only one or two contiguous tissues), while computational models can be far more representative with many complex tissues incorporated in them. The SAR produced in such models from a given source is dependent on several key parameters, including shape and size of the exposed person, the type, location and size of the tissues considered, and their dielectric properties. Most studies assume that the average human is a Caucasian male, only two models of which are common among published results (and thus available for comparison): the Specific Anthropomorphic Mannequin (SAM, a single homogenous tissue model) and the Visible Human (VH, a complex multi-tissue model). Implications for compliance studies are that all members of the general population, including children and females are adequately treated as scaled male adults. Ramifications of such assumptions on SAR compliance are not well described in the current literature.

Generalizations in the literature were often made on the basis of specific models such as Visible Human [1, 2] or MRI data of a handful of human volunteers [3, 4]. Dielectric properties of tissues found in the literature were measured using different equipment, from dead tissue or from animal tissue. Frequently, data from a single subject was used to draw generalized conclusions. The implication is that a randomly chosen individual will provide sufficient information regarding the spread of human anatomical variation, which is unlikely.

A sensitivity study is proposed here to critically assess current assumptions used in SAR studies for compliance and research. Several key parameters will be investigated: what anatomical variations exist in human populations; how these vary between men and women and between children and adults; what effect these variations have on SAR absorption; and how best to model a developing fetus for SAR compliance. It is hoped this study will address many of the problems currently facing SAR assessment.

\section{BACKGROUND}

Assumptions used by current SAR studies are based on sparse and often contradictory data. It is often impossible to compare data due to inconsistencies in models and methodology. 


\section{A. Anatomical variations}

In order to identify how anatomical variations affect SAR, it is important to first establish what variations exist. Data regarding this topic is sparse and inconsistent, rendering impractical comparisons between studies. Cranial thickness in adults is used here as a typical example to demonstrate disparities that exist within the literature.

Using sections excised from four cranial locations frontal and parietal regions, Ross et al. [5] found mean cranial thickness to be between $7.03 \mathrm{~mm}$ and $8.97 \mathrm{~mm}$ in females and between $6.43 \mathrm{~mm}$ and $7.70 \mathrm{~mm}$ in males. They also noted that cranial thickness decreases with age in males and increases in females.

Conversely, Lynnerup found no correlation between cranial thickness and sex, age or general body build [3]. These findings are noted to be in agreement with several earlier studies that found little or no correlation.

Simms and Neely [6] studied variability of temporal bone thickness in children aged 0-20 years of age; their findings also indicate no thickness variability between sexes but displayed large differences between age groups. Adult (20 years old) bone thickness was found to be between about 3 and $7 \mathrm{~mm}$.

\section{B. Dielectric properties of tissues}

Foster et al. [7] details dielectric properties of white and grey brain tissue in dogs for frequencies between $100 \mathrm{MHz}$ and $10 \mathrm{GHz}$. Measurements were taken 8-12 hours after death. No drifts in dielectric properties were noted for several days after death.

A literature review of dielectric tissue properties by Foster and Schwan also points out several studies showing variation related to time after excision was comparable to normal variability in the studied tissues [8].

Gabriel et al. published dielectric properties of various tissues in the frequency range of $10 \mathrm{~Hz}$ to $20 \mathrm{GHz}$ [9]. Samples were from freshly killed animals, human skin and tongues in vivo, and human autopsy material, the latter measured 24-48 hours after death. They report variability in dielectric properties of $\pm 5-10 \%$ above $100 \mathrm{MHz}$ and \pm 15 $25 \%$ at lower frequencies, but also note that variation within species may well exceed variations between species.

Grant et al.'s 1988 study [10] measured dielectric properties of skin at $50 \mathrm{MHz}$ to $2.0 \mathrm{GHz}$ of a male individual at four points on the body. High variability in results was attributed to differences in adipose tissue deposits, presence of sweat ducts and composition of underlying tissue. This study applied a different mathematical analysis technique to the measuring equipment (open-ended coaxial line sensor), which cast doubts on results previously obtained by Grant and others.

It is not known how representative these results are of variance within human populations: insufficient literature is available regarding the comparability of human and animal tissues with regards to dielectric properties. In vivo measurements quoted in the above studies were obtained from a single individual, with no indication as to the possible range.

\section{Human modeling}

Work has been done by several groups to determine the best way to model various anatomical features - for example, Burkhardt and Kuster [11] detail how best to model the human ear in order to represent a reasonable cross-section of mobile phone users. The same group has also published various studies dealing with modeling of SAR in children $[1,12]$.

Drossos et al. [13] investigated the effect human head tissue composition has on electromagnetic energy absorption; they found that a homogeneous model of the human head provided sufficient detail to be accurate when head-equivalent dielectric material was used.

Pregnant females are often modeled as ellipsoid or pear shapes. Chen [14] outlines a more comprehensive model for the uterus complete with uterine wall, fetus and various fetal anatomical features (brain, skeleton) for different developmental stages. This model was based on available information on radiological sensitivity during prenatal period.

Few studies have been performed modeling SAR in human heads at frequencies outside those used by mobile telephones.

\section{METHOD}

Investigations will initially be limited to SAR inside the human head. A review of the current literature will be used to obtain an estimate of variations in a set of key anatomical parameters affecting SAR: tissue dielectric properties, thickness, relative location and tissue size. This information will initially be used to construct models at the $25^{\text {th }}, 50^{\text {th }}$ and $75^{\text {th }}$ percentiles of human anatomical range for each tissue type.

To simplify the problem, complex tissue model (VH) head will at first be modeled varying only five key tissue types: skin, skull, brain, eye and ear. Plane wave excitation will be used as the source. The study may be expanded at a later stage to include more tissues and other forms of excitation.

Each parameter of each tissue type will then be set according to the range found in the literature at the $25^{\text {th }}, 50^{\text {th }}$ and $75^{\text {th }}$ percentile while keeping all other variables and tissues constant. Due to variation in input data and consequent uncertainty of results, it may not be immediately obvious at what level of significance a difference should be set. A difference of ten per cent will initially be set as significant; however this may be modified later based upon results. An a priori rationale may be that it is not useful to 
determine a difference that cannot be easily measured using current compliance techniques.

Mathematical modeling will be performed using a commercially available FDTD (finite-difference time domain) software package. Phantom studies will be included for validation purposes. These will take place at the Telstra Research Laboratories (TRL) using SAM head phantoms; the TRL whole body phantom which contains partitions for head, torso, arms and legs [15]; and an IEEE P1528 compliant flat torso phantom.

Once modeling and validation are completed, those parameters determined to be significant will be combined into a new simplified model that will be analyzed for its efficacy in determining compliance for a range of scenarios.

The scope of this study is limited by its heavy computational load. The frequency range explored will therefore be limited to frequencies used by common sources of public RF exposure such as mobile telephones and other wireless devices in everyday use. If found to be appropriate, contiguous tissue models will be used for optimization. This will allow the use of both simpler models and more efficient modeling codes, for example the hybrid method of moments (MOM) and finite element method (FEM) employed by the commercial product FEKO [16].

\section{Discussion}

The discussion above indicates a need for clearer understanding of how anatomical variations affect SAR absorption.

This study will address at least two key issues that affect SAR investigations: how energy absorption is different between children and adults and between males and females. Both of these issues will concern variations in size, shape, tissue type and distribution and tissue dielectric properties. While these issues, especially that of absorption in adults versus children have previously been studied by others [2, 12], those results are based on the implementation of only one or a few examples of human models. They do not have regard for the full range of variation in the population on relevant parameters, and do not provide any conclusion as to what parameters have the most significant effect on the measured or calculated SAR.

The proposed study will address a number of deficiencies in the current literature. Firstly, current SAR models for children and females mostly use the Caucasian male as basis for tissue parameters such as size and dielectric properties. We need to establish whether this is a viable assumption by ascertaining what differences exist between bodies of males, females and children.

Secondly, we need to define the correlation between age and anatomical variations. This would be done with a view to accomplishing the final part: identifying at what age or size a child may be considered an adult. Literature suggests that there can be more variation within age groups that between them; for example, [6] found that the temporal bone exhibited in some young children was thicker than that of the average adult (20 year old), whereas some 20 year olds had thinner skulls than the average 2 year old.

Another issue considered in this study is the modeling of fetuses for SAR measurement. Gestational female models use some of the same tissue parameters that are used for Caucasian male models, which may not be appropriate. We need to ascertain how dielectric properties and tissue distribution in fetus and mother relate to advancement of pregnancy.

A major challenge we expect to encounter during this study is that we may be unable to distinguish differences in important parameters where the difference is less than the uncertainty in the model. The computationally intensive nature of SAR studies means that it will be necessary to discern what approximations are possible without sacrificing accuracy.

\section{CONCLUSIONS}

A review of existing data has identified a need to more closely examine the assumptions which SAR modeling techniques are based on. This paper outlines a proposed study to critically assess current assumptions underlying SAR testing for compliance and research and postulates potential issues and implications for this work. Expected outcomes of this study are more efficient and accurate models for determining compliance with SAR based exposure levels for a range of RF devices.

\section{REFERENCES}

[1] N. Kuster, A. Christ, N. Chavannes, N. Nikoloski, and J. Frohlich, "Human head phantoms for compliance and communication performance testing of MTE," ISAP i-02, 2002.

[2] O.P. Ghandi, L. Gianluca, and C.M. Furse, "Electromagnetic absorption in the human head and neck for mobile telephones at 835 and $1900 \mathrm{MHz}$," IEEE transactions on microwave theory and techniques, vol. 44, pp. 1884-1897, 1996.

[3] N. Lynnerup, "Cranial thickness in relation to age, sex and general body build in a Danish forensic sample," Forensic Sci. Intl, vol. 117, pp. 45-51, 2001.

[4] U.A. Fill, M. Zankl, N. Petroussi-Henss, M. Siebert, and D. Regulla, "Adult female voxel models of different stature and photon conversion coefficients for radiation protection," health phys., vol. 86, pp. 253272, 2004.

[5] A.H. Ross, R.L. Jantz, and W.F. Mccormick, "Cranial thickness in American females and males," journal of forensic sciences, vol. 43, pp. 267-272, 1998.

[6] D.L. Simms, and J.G. Neely, "Thickness of the lateral surface of the temporal bone in children," ann otol rhinol laryngol, vol. 98, pp. 726$731,1989$.

[7] K.R. Foster, J.L. Schepps, R.D. Stoy, and H.P. Schwan, "Dielectric properties of brain tissue between 0.01 and $10 \mathrm{GHz}$," physics in medicine and biology, vol. 24, pp. 1177-1187, 1979.

[8] K.R. Foster, and H.P. Schwan, "Dielectric properties of tissues and biological materials: A critical review," crit. revs. in biomed. eng., vol. 17, pp. 25-104, 1989.

[9] S. Gabriel, R.W. Lau, and C. Gabriel, "The dielectric properties of biological tissues: Ii. Measurements in the frequency range $10 \mathrm{~Hz}$ to $20 \mathrm{GHz}$," physics in medicine and biology, vol. 41, pp. 2251-2269, 1996. 
[10] J.P. Grant, R.N. Clarke, and N.M. Spyrou, "In vivo dielectric properties of human skin from $50 \mathrm{MHz}$ to $1.0 \mathrm{GHz}$," phys. med. biol., vol. 33, pp. 607-612, 1988 .

[11] M. Burkhardt, and N. Kuster, "Appropriate modeling of the ear for compliance testing of handheld MTE with SAR safety limits at 900/1800 MHz," IEEE transactions on microwave theory and techniques, vol. 48, pp. 1927-1935, 2000.

[12] F. Schonborn, M. Burkhardt, and N. Kuster, "Difference in energy absorption between heads of adults and children in the near field of sources," health phys., vol. 74, pp. 160-168, 1998.

[13] A. Drossos, V. Santomaa, and N. Kuster, "The dependence of electromagnetic energy absorption upon human head tissue composition in the frequency range of $300-3000 \mathrm{mhz}, "$ IEEE transactions on microwave theory and techniques, vol. 48, pp. 19881995, 2000.

[14] J. Chen, "Mathematical models of the embryo and fetus for use in radiological protection," health phys., vol. 86, pp. 285-295, 2004.

[15] R. Mcintosh, R. Mckenzie, and A. Carratelli, "The numerical evaluation of a SAR measurement phantom at the telstra research laboratories," presented at URSI Wireless Applications of Radio Science Workshop (WARS 02), Sydney, Australia, 2001.

[16] Www.Feko.Info, "Feko," 2004. 